\title{
Embolia pulmonar en el Servicio de Emergencias: epidemiología, riesgo y abordaje diagnóstico
}

\section{Dario Eduardo García*}

Servicio de Admisión Paciente Crítico, Hospital El Cruce Alta Complejidad en Red Dr. Néstor Carlos Kirchner, Florencio Varela, Buenos Aires, Argentina

\section{Resumen}

En la actualidad la embolia pulmonar constituye un problema de salud pública que requiere asistencia cardiovascular inmediata, debido a que constituye una de las principales causas de mortalidad de origen cardiovascular. La detección de esta patología representa un verdadero desafío en los Servicios de Emergencias (SE), dado por su enfoque de diagnóstico complejo, la inespecificidad de los síntomas y que puede estar enmascarada por otras patologías. La utilización de forma combinada de las diferentes herramientas diagnosticas permitirá hacer un diagnóstico precoz, con el consiguiente manejo eficiente del paciente.

Palabras clave: Embolismo pulmonar. Insuficiencia respiratoria. Epidemiología. Diagnóstico. Servicio de Emergencias.

\section{Pulmonary embolism in the Emergency Department: epidemiology, risk and diagnostic approach}

\begin{abstract}
Currently, pulmonary embolism is one of the main causes of cardiovascular mortality and considered a public health problem that requires immediate cardiovascular assistance. The detection of pulmonary embolism represents a real challenge for Emergency Services (ES), given by its complex diagnostic approach, the no distinctive symptoms, and the possibility to be masked by other diseases. The combined use of different diagnostic tools will allow its early diagnosis, with the consequent efficient management of the patient.
\end{abstract}

Key words: Pulmonary embolism. Respiratory Insufficiency. Epidemiology. Diagnosis. Emergency services.

\section{Introducción}

La embolia pulmonar (EP) es un problema de salud pública y que requiere asistencia cardiovascular inmediata, es la tercera causa más frecuente de urgencia vascular después de la cardiopatía isquémica o el ictus ${ }^{1,2}$.
La EP es una de las manifestaciones de la tromboembolia venosa (TEV), al igual que de la trombosis venosa profunda $^{3}$ (TVP) y representa un verdadero desafío en los Servicios de Emergencias (SE), dada por la gran variedad de síntomas que puede presentar y que, además, puede estar enmascarada por otras patologías ${ }^{4,5}$.

\section{Correspondencia:}

*Dario Eduardo García

Av. Calchaquí, 5401, Florencio Varela Fecha de recepción: 17-02-2019

C.P. 1888, Buenos Aires, Argentina Fecha de aceptación: 10-04-2019

E-mail: darioabue@yahoo.com.ar_ DOI: 10.24875/REIE.19000017
Disponible en internet: 20-06-2019 Rev Educ Investig Emer. 2019;1(2):66-76 www.medicinadeemergencias.com 2604-6520 @ 2019 Sociedad Mexicana de Medicina de Emergencias, AC. Publicado por Permanyer México SA de CV. Este es un artículo Open Access bajo la licencia CC BY-NC-ND (http://creativecommons.org/licenses/by-nc-nd/4.0/). 
Tabla 1. Factores de riesgo

\begin{tabular}{|c|c|c|}
\hline \multicolumn{3}{|l|}{ Factores de riesgo } \\
\hline Factores hereditarios & Factores adquiridos & Factores probables \\
\hline $\begin{array}{l}\text { - Déficits antitrombina } \\
\text { - Déficits proteína C } \\
\text { - Déficits proteína S } \\
\text { - Mutaciones del factor V } \\
\text { - Mutación gen protrombina 20210A } \\
\text { - Déficits plasminógeno } \\
\text { - Síndrome de plaqueta "pegajosa" } \\
\text { - Aumento lipoproteína a }\end{array}$ & $\begin{array}{l}\text { - Síndrome antifosfolipídico } \\
\text { - Policitemia vera } \\
\text { - Obesidad } \\
\text { - Trauma raquimedular } \\
\text { - Movilización reducida } \\
\text { - Cirugía mayor } \\
\text { - Cirugía ortopédica mayor } \\
\text { - Embarazo y puerperio } \\
\text { - Edad avanzada } \\
\text { - Cáncer } \\
\text { - Viajes prolongados } \\
\text { - Fibrilación auricular } \\
\text { - Valvulopatía } \\
\text { - Anticonceptivos orales } \\
\text { - Síndrome de HIT (trombocitopenia inducida por heparina) }\end{array}$ & $\begin{array}{l}\text { - Hiperhomocisteinemia } \\
\text { - Factores VIII-IX-XI elevados } \\
\text { - Fibrinogenemia elevada }\end{array}$ \\
\hline
\end{tabular}

Adaptada de Di Nisio, et al., 20163; García-Frade, et al., 2016³; Tapson, et al., $2012^{14}$.

La EP es una causa importante de morbimortalidad en la fase aguda y presenta complicaciones crónicas graves, como la hipertensión pulmonar (HTP) tromboembólica crónica $(1.5 \%)^{4,6}$, el síndrome postrombótico y la recurrencia de EP ( $8 \%$ a los 6 meses) $)^{4,6}$.

\section{Definición}

La EP es el obstrucción parcial o total en las arterias pulmonares por material trombótico (émbolo) originado en cualquier segmento del sistema venoso, principalmente el profundo de lo miembros inferiores (80-95\% $)^{1,6}$.

\section{Epidemiología}

La epidemiologia de la EP es difícil de determinar, ya que se presenta de forma muy variada, desde la forma asintomática hasta la forma más grave de presentación, que es la muerte súbita 4 .

En los EE.UU. se estiman unas 500,000-600,000 personas con EP/año ${ }^{7}$, en Francia 100,000 personas/año y en Gran Bretaña 65,000 personas/año.

En la población europea se estima una incidencia de TVP de 70-140 casos/100,000 persona-año ${ }^{8,9}$. En EE.UU. la incidencia estimada es de 100 a 200 casos por cada 100,000 personas-año $0^{7,8}$. En Latinoamérica no se han encontrado datos fiables de su incidencia.

Su incidencia aumenta con la edad, es 8 veces más frecuente en los mayores de 80 años que en los menores de 50 años $^{9-11}$. Respecto al sexo, las mujeres se afectan con mayor frecuencia a edades más tempranas; pero esta relación se invierte en los ancianos ${ }^{9}$. La incidencia es similar en los negros, pero menor en los asiáticos ${ }^{9}$.

\section{Factores de riesgo y fisiopatología}

Los factores de riesgo son aquellas condiciones inherentes del paciente (permanentes) o situaciones clínicas (temporales) que se asocian con un aumento de la incidencia de $\mathrm{EP}^{7,10-12}$. Los factores más importantes $^{13,14}$ se pueden apreciar en la tabla 1.

La patogenia de la TVP tiene su origen en la clásica triada de Virchow descrita en 1856: lesión en las paredes vasculares, estasis venosa y estados de hipercoagulabilidad sanguínea $4,7,12$.

Los factores de riesgos (tanto adquiridos como congénitos) están presentes en aproximadamente el $75 \%$ de los casos (se describen en la Tabla 2).

La EP aguda interfiere con el intercambio gaseoso y la circulación, el compromiso hemodinámico será más grave cuanto más proximal sea su localización e impacto, cuanto mayor grado de obstrucción presente e inversamente proporcional a la reserva cardiopulmonar ${ }^{4}$. La presión arterial pulmonar aumenta ante la obstrucción del lecho arterial pulmonar, generando aumento de la resistencia vascular pulmonar aguda, dilatación aguda, isquemia y disfunción del ventrículo derecho (VD), por su escasa capacidad de adaptación. Posteriormente tiene lugar el compromiso del ventrículo izquierdo, disminución del gasto cardíaco y finalmente la inestabilidad hemodinámica y choque ${ }^{4}$. 
Tabla 2. Factores de riesgo para tromboembolia pulmonar

\begin{tabular}{|c|c|c|}
\hline $\begin{array}{l}\text { Factores de riesgo fuerte (índice de } \\
\text { probabilidades }>10 \text { ) }\end{array}$ & $\begin{array}{l}\text { Factores de riesgo moderado (índice de } \\
\text { probabilidades 2-9) }\end{array}$ & $\begin{array}{l}\text { Factores de riesgo débil (índice de } \\
\text { probabilidades <2) }\end{array}$ \\
\hline $\begin{array}{l}\text { - Fractura de extremidad inferior } \\
\text { - Hospitalización por insuficiencia cardíaca } \\
\text { o fibrilación/aleteo auricular (en los } 3 \\
\text { meses previos) } \\
\text { - Reemplazo de cadera o rodilla } \\
\text { - Traumatismo importante } \\
\text { - Infarto de miocardio (en los } 3 \text { meses } \\
\text { previos) } \\
\text { - Tromboembolia venosa previa } \\
\text { - Lesión de medula espinal }\end{array}$ & $\begin{array}{l}\text { - Cirugía artroscópica de rodilla } \\
\text { - Enfermedades autoinmunitarias } \\
\text { - Transfusión de sangre } \\
\text { - Vías venosas centrales } \\
\text { - Quimioterapia } \\
\text { - Insuficiencia cardíaca congestiva o } \\
\text { respiratoria } \\
\text { - Agentes estimuladores de la eritropoyesis } \\
\text { - Terapia de reemplazo hormonal (depende } \\
\text { de la formulación) } \\
\text { - Fertilización in vitro } \\
\text { - Infección (específicamente neumonía, } \\
\text { infección del tracto urinario y VIH) } \\
\text { - Enfermedad inflamatoria intestinal } \\
\text { - Cáncer (mayor riesgo en enfermedad } \\
\text { metastásica) } \\
\text { - Anticonceptivos orales } \\
\text { - Accidente cerebrovascular paralítico } \\
\text { - Periodo posparto } \\
\text { - Trombosis venosa superficial } \\
\text { - Trombofilia }\end{array}$ & $\begin{array}{l}\text { - Reposo en cama > } 3 \text { días } \\
\text { - Diabetes mellitus } \\
\text { - Hipertensión } \\
\text { - Inmovilidad por estar sentado (p. ej.., } \\
\text { viaje prolongado en coche o avión) } \\
\text { - Edad en aumento } \\
\text { - Cirugía laparoscópica (p. ej., } \\
\text { colecistectomía) } \\
\text { - Obesidad } \\
\text { - Gestación } \\
\text { - Venas varicosas }\end{array}$ \\
\hline
\end{tabular}

VIH: Virus de la inmunodeficiencia humana. Adaptada de Konstantinides, et al., 20144.

\section{Clasificación}

La clasificación clínica actual de la gravedad de un episodio agudo de EP se basa en estimar el riesgo de muerte precoz, definido por la mortalidad intrahospitalaria y a los 30 días $^{4}$.

Esta estratificación tiene importantes implicaciones para las estrategias tanto diagnosticas como terapéuticas, basándose en el estado clínico del paciente en el momento de la presentación 4 .

Se clasifica en tres grupos:

- Alto riesgo. Presenta choque (definido como presión arterial sistólica $<90 \mathrm{mmHg}$ o una caída de presión sistólica $\geq 40 \mathrm{mmHg}$ durante más de 15 min, si no la causan una arritmia de nueva instauración, hipovolemia o sepsis ${ }^{4,15-17}$.

- Riesgo intermedio. Paciente sin choque pero con pruebas de necrosis miocárdica o sobrecarga del VD (elevación de troponina o dímero D), cambios electrocardiográficos o signos en las pruebas de diagnóstico por la imagen (ecocardiografía o tomografía computarizada) de dilatación o disfunción del VD; elevación de las presiones pulmonares y/o compresión del tabique interventricular ${ }^{4,15-17}$.

- Riesgo bajo. Pacientes sin los criterios anteriores ${ }^{4,15-17}$.

\section{Anamnesis}

La anamnesis debe ser orientada y dirigida a la situación clínica del paciente. El reconocimiento de los factores de riesgo, los antecedentes patológicos, y los signos/síntomas de EP permitirá hacer un diagnóstico precoz, con el consiguiente manejo óptimo del paciente. En la anamnesis entre el 10 y el $35 \%$ de los pacientes informan antecedente de $\mathrm{EP}^{7}$.

\section{Cuadro clínico}

Muchas veces, los pacientes presentan manifestaciones clínicas inespecíficas y poco sensibles. Pueden manifestarse desde síntomas leves y de difícil de diagnóstico hasta manifestaciones graves. Se debe sospechar ante disnea de nueva aparición, empeoramiento de su disnea habitual, dolor torácico, síncope o hipotensión sin una clara explicación alternativa.

La disnea, la taquipnea y la taquicardia son los signos más frecuentes. El síncope ${ }^{18-20}$ es más frecuente cuando la EP es masiva, lo que significa que obstruye más de la mitad de la circulación pulmonar ${ }^{18,19}$.

El dolor torácico puede ser pleurítico (40-48\%), que es causado por irritación pleural debida a émbolos distales que causan infarto pulmonar ${ }^{4,18-20}, 0$ dolor torácico 
Tabla 3. Síntomas/signos más frecuentes de embolia pulmonar (EP)

\begin{tabular}{|l|c|l|c|}
\hline \multicolumn{2}{|l|}{ Síntomas/signos más frecuentes de EP } & \multicolumn{2}{l|}{ Prevalencia } \\
\hline Síntomas & Prevalencia & Signos & $68-70 \%$ \\
\hline Disnea & $72-80 \%$ & Taquipnea & $40 \%$ \\
\hline Dolor torácico pleurítico & $52-59 \%$ & Rales pulmonares & $24 \%$ \\
\hline Tos & $20-36 \%$ & Taquicardia & $13 \%$ \\
\hline Hemoptisis & $8-11 \%$ & $4 .^{\circ}$ ruido cardíaco $\left(s_{4}\right)$ & $12-19 \%$ \\
\hline Palpitaciones & $18 \%$ & Síncope & $7-12 \%$ \\
\hline & & Fiebre & $15 \%$ \\
\hline
\end{tabular}

TVP: Trombosis venosa profunda.

Adaptada de Mcgee, 2018 ${ }^{18}$, Renier, et al., 2018 ${ }^{19}$; Morice, et al., 200020; Stein, et al., $2007^{54}$.

de carácter anginoso (por isquemia del VD), que requiere un diagnóstico diferencial con el síndrome coronario agudo $(\mathrm{SCA})^{4}$.

Los síntomas más frecuentes de EP se describen en la tabla 3.

\section{Diagnóstico}

La estrategia óptima para el diagnóstico de la EP entre los expertos sigue siendo controvertido y puede diferir según variables como el entorno de atención (urbano, rural), que puede comprometer el acceso a los estudios complementarios ${ }^{21}$. Además, ninguna prueba aislada es lo suficientemente sensible y específico para confirmar o descartar la presencia de EP. Por ello se debe tener en cuenta la utilización de forma combinada de las diferentes herramientas diagnósticas (anamnesis, sospecha clínica, examen físico, reglas de decisión clínica y estudios complementarios).

Los diagnósticos diferenciales más frecuentes a tener en cuenta son: edema pulmonar cardiogénico, neumonía aguda de la comunidad, exacerbación de la enfermedad pulmonar obstructiva crónica y asma aguda ${ }^{22}$.

\section{Estrategias diagnósticas}

Se sugiere que la elección de las pruebas diagnósticas se haga en relación con la probabilidad clínica y situación hemodinámica del paciente.

En los últimos años se han desarrollado diferentes estrategias y múltiples reglas de decisión clínica (RDC) ${ }^{5,16}$, cuyos objetivos en los pacientes ambulatorios de bajo-moderado riesgo de presentar EP son: a) minimizar la necesidad de tomografía computarizada de las arterias pulmonares (TCAP), b) reducir la exposición del paciente a la radiación, c) reducir los costos y d) reducir el tiempo de evaluación ${ }^{2,12,23-26}$.

Las RDC se dividen en:

- Escalas de valoración del riesgo ${ }^{5}$. Calculan el riesgo de que un paciente tenga un evento tromboembólico venoso.

- Escalas de probabilidad clínica ${ }^{5}$. Evalúan que un determinado paciente presente ese evento; las principales son:

- Escala de Wells ${ }^{27,28}$ y Escala de Wells simplifica$\mathrm{da}^{29}$ (Tabla 4).

- Escala de Ginebra ${ }^{30}$ y Escala revisada de Ginebra $^{31}$ (Tabla 4).

- Escala PERC ${ }^{32-34}$ (Pulmonary Embolism Ruleout Criteria) (Tabla 5).

- Escalas pronósticas ${ }^{5}$. Estiman el riesgo de muerte una vez que se ha establecido el diagnóstico.

- Escalas de probabilidad de recurrencia ${ }^{5}$. Valoran el riesgo de volver a presentar un nuevo episodio una vez finalizado el tratamiento.

En los SE las escalas de Wells y de Ginebra son las más utilizadas y recientemente se han simplificado en un intento de aumentar su uso $0^{5,35}$.

Por otra parte, con fines de tratamiento clínico, se define «EP confirmada» como una probabilidad de EP lo suficientemente alta para indicar la necesidad de un tratamiento específico de EP, y «EP excluida» como una probabilidad de EP lo suficientemente baja para justificar la retirada del tratamiento específico de EP con un riesgo aceptablemente bajo 4 . 
Tabla 4. Escalas de probabilidad clínica más utilizadas en los servicios de emergencias

\begin{tabular}{|c|c|c|c|c|c|}
\hline Criterio & $\begin{array}{l}\text { Puntuación } \\
\text { Wells }{ }^{27,28}\end{array}$ & $\begin{array}{c}\text { Puntuación Wells } \\
\text { modificada }\end{array}$ & Criterio & $\begin{array}{c}\text { Puntuación } \\
\text { de } \\
\text { Ginebra }^{30}\end{array}$ & $\begin{array}{c}\text { Puntuación de } \\
\text { Ginebra } \\
\text { modificada }^{31}\end{array}$ \\
\hline Factores de riesgo & Puntaje & Puntaje & Factores de riesgo & Puntaje & Puntaje \\
\hline $\begin{array}{l}\text { - Síntomas clínicos de TVP } \\
\text { - Otro diagnóstico menos } \\
\text { probable que EP } \\
\text { - Frecuencia cardíaca } \\
\text { mayor a } 100 \text { Ipm } \\
\text { - Inmovilización de más de } \\
3 \text { días o cirugía en las } \\
4 \text { semanas previas } \\
\text { - TVP o EP previo } \\
\text { - Hemoptisis } \\
\text { - Cáncer Activo }\end{array}$ & $\begin{array}{c}3 \\
3 \\
1.5 \\
1.5 \\
1.5 \\
1 \\
1\end{array}$ & $\begin{array}{l}1 \\
1 \\
1 \\
1 \\
1 \\
1 \\
1\end{array}$ & $\begin{array}{l}\text { - Edad mayor de } 65 \text { años } \\
\text { - TVP o EP previo } \\
\text { - Cirugía o fractura en el mes } \\
\text { previo } \\
\text { - Cáncer activo } \\
\text { - Dolor de extremidad inferior } \\
\text { unilateral } \\
\text { - Hemoptisis } \\
\text { - Frecuencia cardíaca de } 75 \\
\text { a } 94 \text { Ipm } \\
\text { - Frecuencia cardíaca mayor } \\
\text { o igual a } 95 \text { Ipm } \\
\text { - Dolor a la palpación venosa } \\
\text { profunda del miembro } \\
\text { inferior y edema unilateral }\end{array}$ & $\begin{array}{l}1 \\
3 \\
2 \\
2 \\
3 \\
2 \\
3 \\
5 \\
4\end{array}$ & $\begin{array}{l}1 \\
1 \\
1 \\
1 \\
1 \\
1 \\
1 \\
2 \\
1\end{array}$ \\
\hline Probabilidad clínica & Puntaje & Puntaje & Probabilidad clínica & Puntaje & Puntaje \\
\hline Alto & $>6$ & $\begin{array}{c}>2 \\
\text { (probabilidad alta) }\end{array}$ & Alto & $>11$ & $\begin{array}{c}>2 \\
\text { (probabilidad alta) }\end{array}$ \\
\hline Intermedio & $2-6$ & --------- & Intermedio & $4-10$ & ------ \\
\hline Bajo & $0-1$ & $\begin{array}{c}0-1 \\
\text { (probabilidad baja) }\end{array}$ & Bajo & $0-3$ & $\begin{array}{c}0-1 \\
\text { (probabilidad } \\
\text { baja) }\end{array}$ \\
\hline
\end{tabular}

EP: embolia pulmonar: TVP: trombosis venosa profunda.

\section{Estudios complementarios}

\section{Dímero D}

Es el biomarcador más utilizado. Es producto de la degradación de la fibrina y está elevado en presencia de trombosis aguda, a causa de la activación simultánea de la coagulación y la fibrinólisisis ${ }^{4,23,35,36}$. El valor predictivo negativo (VPN) del dímero $\mathrm{D}$ es alto, y un valor normal de dímero $D$ hace que sea improbable la $\mathrm{EP}^{4,23,35,36}$. Pero una amplia variedad de afecciones producen aumento plasmático de fibrina, como en los pacientes hospitalizados, el cáncer, la inflamación, la hemorragia, el traumatismo, la cirugía y la necrosis; en consecuencia, el valor predictivo positivo (VPP) de altos valores de dímero $D$ es bajo y el estudio de este no es útil para la confirmación de $\mathrm{EP}^{4,23,35,36}$.

\section{Enzyme linked immunosorbent assay}

EI ELISA (enzyme linked immunosorbent assay) cuantitativo $u$ otros análisis derivados de este tienen una sensibilidad diagnóstica del $95 \%$ o superior, por lo que pueden utilizarse para excluir la EP en pacientes con una probabilidad previa a la prueba baja o mode$\operatorname{rada}^{4,9,23,35-37}$. La especificidad del dímero $\mathrm{D}$ disminuye con la edad, hasta casi un $10 \%$ en pacientes mayores de 80 años ${ }^{4,36}$; el uso de puntos de corte ajustados por edad mejora el rendimiento del estudio del dímero $D$ en ancianos $^{4,36}$. Valores de corte ajustados por edad (edad $\times 10 \mu \mathrm{g} / \mathrm{l}$ por encima de los 50 años) permitieron aumentar la especificidad del 34 al $46 \%$ conservando una sensibilidad superior al 97\% 4,23,36,37.

En la revisión sistemática y metaanálisis (RS-MA) de Crawford, et al. ${ }^{23}$ concluyeron que las pruebas cuantitativas del dímero $\mathrm{D}$ utilizadas en los SE tienen pocos resultados falsos negativos, pero niveles muy altos de resultados falsos positivos, por lo que la prueba es útil para descartar EP, pero un resultado positivo requiere de la realización de pruebas de imagenología ${ }^{23}$.

\section{Radiografía de tórax}

Es un estudio de amplia disponibilidad y bajo costo. Se utiliza como herramienta para excluir diagnósticos diferenciales (neumotórax, neumonía, insuficiencia cardíaca) $)^{10,38}$. 
Tabla 5. Regla de PERC

\section{Regla de PERC}

- Edad menor a 50 años

- Frecuencia cardíaca menor a $100 \mathrm{lpm}$

- Saturación de oxígeno $\geq 95 \%$

- Sin hemoptisis

- No uso de estrógenos

- Sin TVP o EP previo

- No hay edema unilateral en miembros inferiores

- No hay cirugía o trauma que requiriesen hospitalización en el último mes

- Negativo. Si cumplen los 8 criterios, muy baja probabilidad de tener EP, no se requieren pruebas adicionales.

- Positivo. Todos los demás pacientes deberán ser considerados para realizar pruebas adicionales como dímero D o imágenes.

EP: embolia pulmonar; PERC: Pulmonary Embolism Rule-out Criteria; TVP: trombosis venosa profunda.

Adaptada de Kline, et al., 2004²; Carpenter, et al., 200933; Kline, et al., 2013 ${ }^{34}$

En el estudio ICOPER (International Cooperative Pulmonary Embolism Registry) ${ }^{39}$, la radiografía fue anormal en el $75 \%$ de los pacientes evaluados, con hallazgos muy inespecíficos. Entre los signos más frecuentes debemos destacar: incremento de la silueta cardíaca $(27 \%)$, derrame pleural $(23 \%)$, elevación de un hemidiafragma (20\%), aumento del diámetro de la arteria pulmonar (19\%) y atelectasia (18\%).

\section{Ecografía pulmonar}

La ecografía se desarrolló a partir del sonar, creado en la Primera Guerra Mundial, con las primeras aplicaciones en el ser humano en el año 1947; en 1958 se realizaron las primeras aplicaciones en las patologías abdominales y finalmente su aplicación clínica pulmonar ocurrió en la década de los noventa ${ }^{40}$.

En la evaluación del paciente con disnea en el SE, la ecografía pulmonar sirve para complementar a la anamnesis y a la exploración física. Permite orientar el diagnóstico etiológico y realizar el diagnóstico en muchos pacientes. Incluso, a veces, antes de disponer de pruebas complementarias como la radiografía de tórax o los estudios de laboratorio ${ }^{41}$.

Permite evaluar desde:

- La pared torácica y su musculatura ${ }^{40}$.

- Descartar patología diafragmática ${ }^{40}$.

- Descartar patología pleural ${ }^{40}$ (como el derrame pleural, con una sensibilidad del $100 \%$ y especificidad del $99 \%$, y el neumotórax, con una sensibilidad del $85-100 \%$ y especificidad del $83-99 \%)^{40}$.

- Descartar patología parenquimatosa ${ }^{42}$ :

- EP, descrito por primera vez en $1971^{41}$. La imagen ecográfica variará, según en qué fase se encuentre (precoz, establecida y de resolución). Las lesiones características son:
- Lesiones (habitualmente 20 3) muy próximas a la pleura, móviles con la respiración y de bordes bien definidos, de $1-1.5 \mathrm{~cm}$ de tamaño.

- Asociadas con derrame pleural subyacente.

- Y con ausencia de señal Doppler por la inexistencia de flujo arterial.

Para el diagnóstico se requiere al menos dos de estas lesiones.

- Atelectasias, colapso pulmonar, neumonía, patrón vascular, consolidación parenquimatosa, atelectasias $^{42}$.

Una forma de sistematización de la evaluación pulmonar es por medio del protocolo BLUE (Bedside Ultrasound in Emergency) $)^{43,44}$ y el protocolo FALLS

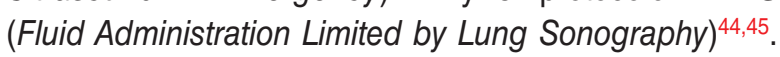

La RS-MA de Squizzato ${ }^{46}$ evaluó la precisión diagnóstica de la ecografía pulmonar para el diagnóstico de EP. Incluyó 10 estudios, 887 pacientes. La sensibilidad fue del $87 \%$ (Intervalo de confianza del 95\% [IC 95\%] 79-92\%) y la especificidad del 82\% (IC 95\%: $71-89 \%)$. Se concluyó que la ecografía pulmonar puede ser una herramienta de diagnóstico útil para los pacientes con sospecha de EP.

La RS-MA de Jiang ${ }^{47}$ también evaluó la precisión diagnóstica de la ecografía pulmonar para el diagnóstico de EP. Incluyó 13 estudios, 1,356 pacientes. La sensibilidad fue del $85 \%$ (IC 95\%: 78-90\%) y la especificidad del 83\% (IC 95\%: 73-90\%). Se concluyó que la ecografía pulmonar es una herramienta de diagnóstico útil para el diagnóstico de sospecha de EP.

\section{Ecocardiograma}

\section{ECOCARDIOGRAMA TRANSTORÁCICO}

El ecocardiograma es casi indispensable en la evaluación de un paciente con EP, ya que permite 
sospechar el diagnóstico ante el hallazgo de dilatación del VD o existencia de HTP.

Este método tiene un bajo VPN (40-50\%), por lo que un resultado negativo no puede excluir EP4. Por otro lado, en ausencia de EP aguda también se puede encontrar signos de sobrecarga o disfunción del VD, que pueden deberse a enfermedad cardíaca o respiratoria concomitante $4,10,11$.

Los hallazgos ecocardiográficos más importantes son la dilatación del VD (25\%) y la disfunción del VD (que se produce en la mayor parte del choque secundario a la EP), por lo que su detección es útil para la estratificación de riesgos de la enfermedad ${ }^{4,10,11}$. Otros hallazgos son: presencia de HTP sistólica (velocidades pico de insuficiencia tricúspidea de entre 3 y $3.6 \mathrm{~m} / \mathrm{s})^{10,11}$, patrón de eyección del VD alterado («signo de 60-60») o en la contractilidad disminuida de la pared libre del VD en comparación con el vértice del VD («signo de McConnell»).

El examen ecocardiográfico no está recomendado como parte de las pruebas diagnósticas para pacientes normotensos hemodinámicamente estables con sospecha de EP (no de alto riesgo) ${ }^{4}$. Esto es contrario a la sospecha de EP de alto riesgo, en la que la ausencia de signos ecocardiográficos de sobrecarga o disfunción del VD prácticamente excluye la EP como causa de inestabilidad hemodinámica ${ }^{4,10,11}$.

\section{ECOCARDIOGRAMA TRANSESOFÁGICO}

Su utilidad radica en la posibilidad de detectar trombos en el tronco o las ramas proximales de arterias pulmonares cuando la tomografía no está disponible y en especial en pacientes ventilados ${ }^{10,11}$. Es un estudio de segunda elección y de gran aporte cuando no se dispone de otra información confirmatoria. Permite además descartar otras patologías cardiovasculares como disección aórtica o insuficiencia mitral aguda ${ }^{10,11}$.

\section{Angiografía pulmonar por tomografía computarizada de tórax}

Desde la introducción de la angiografía con TC multidetectores (TCMD), se ha convertido en el método de elección para el diagnóstico de EP4. En pacientes con probabilidad clínica baja o intermedia de EP, valorada por la puntuación en la escala de Wells, una TC negativa tenía un alto VPN (96-89\%), mientras en quienes tenían una probabilidad alta era de solo el $60 \%$. Por el contrario, el VPP de una TC positiva era alto (92-96\%) en pacientes con probabilidad clínica intermedia 0 alta, pero mucho menor (58\%) en pacientes con baja probabilidad previa a la prueba de EP4.

La RS-MA de Trujillo Santos, et al. ${ }^{48}$ (10 estudios, 2,288 pacientes), concluyeron que los pacientes con disfunción del VD presentaron mayor mortalidad global (Odds Ratio [OR] 1.8 IC 95\% 1.3-2.6) y mayor mortalidad relacionada con la EP (OR: 2.4; IC 95\%: 1.2-4.7).

\section{Centellograma pulmonar de ventilación/ perfusión}

Al ser un procedimiento con menos radiación y medio de contraste, la exploración de V/Q puede aplicarse preferentemente en pacientes ambulatorios con baja probabilidad clínica y una radiografía de tórax normal, pacientes jóvenes (particularmente mujeres), gestantes, con historia de anafilaxia inducida por medios de contraste y fuerte historia alérgica, con insuficiencia renal grave o con mieloma y paraproteinemia ${ }^{4}$.

Para facilitar la comunicación, es preferible una clasificación de tres niveles: gammagrafía $\mathrm{V} / \mathrm{Q}$ normal (excluye la EP), gammagrafía $V / Q$ de alta probabilidad (es considerado como diagnóstico confirmado de EP en los pacientes con probabilidad clínica intermedia-alta) y no diagnóstico ${ }^{4}$ o no concluyente, en la que se recomienda pruebas diagnósticas adicionales ${ }^{10,11}$.

\section{Eco-Doppler en la trombosis venosa profunda}

El diagnóstico de TVP es una manera indirecta para determinar la posibilidad de $\mathrm{EP}^{1,10,11}$. En presencia de EP, la TVP es positiva en el 50 al $70 \%$ de los casos ${ }^{1,10,11}$. Además, aproximadamente el $50 \%$ de los pacientes con TVP presentan evidencia de EP asintomática ${ }^{1,10,11}$. En los pacientes con TVP y EP clínica, la presencia de trombosis pulmonar se confirma en el $90 \%$ de los casos ${ }^{1,10,11}$.

El eco-Doppler venoso de los miembros inferiores es el método diagnóstico más útil, fácil y accesible para arribar al diagnóstico de TVP1,10,11. La presencia de una vena no colapsable constituye el pilar confirmatorio, con una sensibilidad mayor del $90 \%$ y una especificidad del 95\% para el diagnóstico de trombosis proxi$\mathrm{mal}^{1,10,11}$. Sin embargo, un eco-Doppler venoso de los miembros inferiores es normal en un $30-50 \%$ de los pacientes con enfermedad de Parkinson (EP); el método no excluye entonces el diagnóstico de TEP (aunque reduce su probabilidad) $)^{1,10,11}$. La ausencia de TVP determinada por eco-Doppler se acompaña de un riesgo menor de recurrencia de TEP ${ }^{1,10,11}$. 


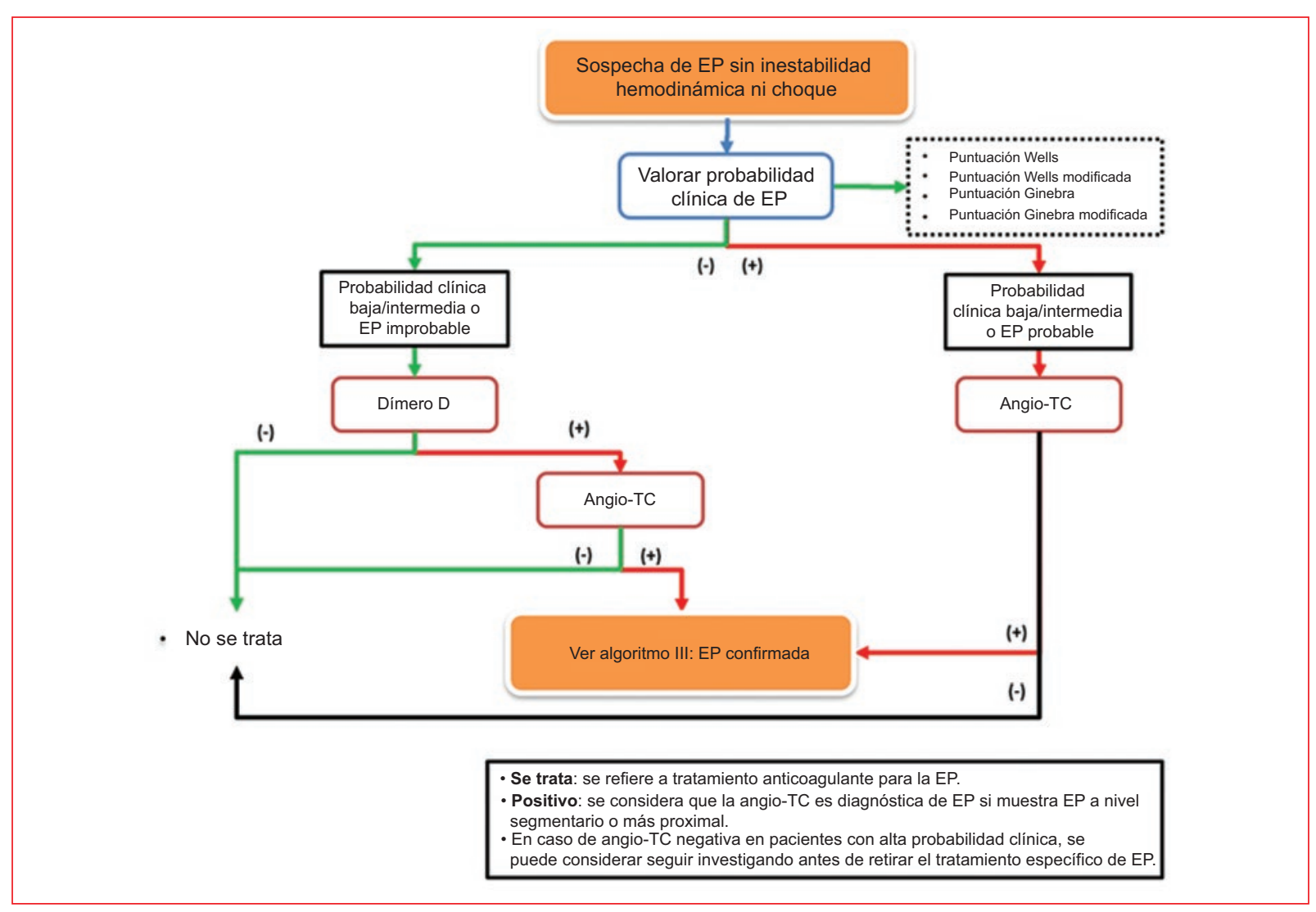

Figura 1. Algoritmo I. Diagnóstico de sospecha de embolia de pulmón sin inestabilidad hemodinámica o choque (adaptada de Konstantinides, et al., 2014\%).

EP: embolia pulmonar; Angio-TC: angiotomografía computarizada.

\section{Resonancia magnética nuclear de tórax}

La RS-MA de Zhou, et al. ${ }^{49}$ evaluó el rendimiento diagnóstico de la RMN en la EP aguda (15 estudios); presentó una sensibilidad del 84\% (IC 95\%: 80-87) y una especificidad del 97\% (IC 95\%: 96-98), concluyó que la RMN proporciona una alta precisión diagnóstica para la detección de EP aguda. La RS-MA de Zhou, et al. ${ }^{50}$ (10 estudios, 590 pacientes) concluyó que tiene una mejor sensibilidad y especificidad para detectar EP de arteria subsegmentaria comparada con la TC, sensibilidad de la TC del 90\% (IC 95\%: 85-93), especificidad del 88\% (IC 95\%: 77-95), mientras con la RMN fue del $92 \%$ (IC 95\%: 89-94) y el 91\% (IC 95\%: 77-97), respectivamente.

\section{Algoritmos de diagnóstico}

El uso de algoritmos diagnósticos está justificado y se han propuesto y validado diversas combinaciones de valoración clínica, determinación de dímero $D$ en plasma y pruebas de imagen. Estas estrategias se estudiaron en pacientes que se presentaron con sospecha de EP en los SE durante la estancia en el hospital ${ }^{4}$ y la estrategia diagnostica a desarrollar se basará en la estabilidad hemodinámicamente del paciente (Fig. 1). En un paciente muy inestable, la evidencia ecocardiográfica de disfunción del VD es suficiente motivo para la reperfusión inmediata sin más pruebas ${ }^{4}$ (Fig. 2).

\section{Evaluación económica}

El estudio de Sinclair, et al. ${ }^{21}$ concluyó que el diagnóstico de los pacientes con EP era rentable si la disposición a pagar fue de \$ (dólares) 1,481 por Quality Adjusted Life Year (QALY). La estrategia diagnóstica con tomografía de tórax (TCT) fue más rentable, ya que se encontró que esta tenía mayor precisión, menor proporción de hallazgos no diagnósticos y estaba asociada con los costos más bajos.

El uso de RDC para determinar la probabilidad clínica previa de la EP y la aplicación de la prueba de 


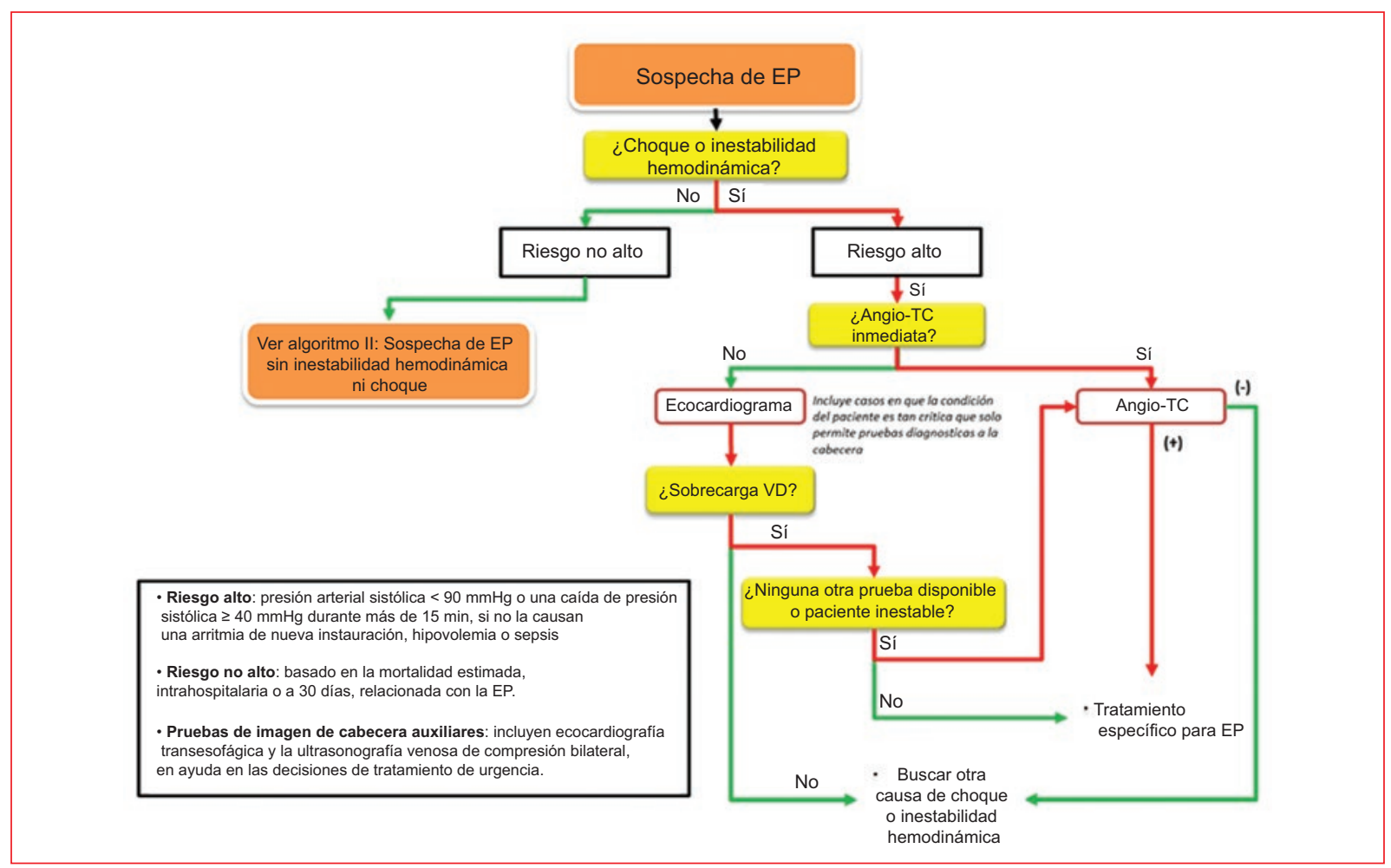

Figura 2. Algoritmo II. Diagnóstico de sospecha de embolia de pulmón con inestabilidad hemodinámica o choque (adaptada de Konstantinides, et al., 20144).

EP: embolia pulmonar; Angio-TC: angiotomografía computarizada; VD: ventrículo derecho.

descarte en pacientes con riesgo de EP bajo a moderado puede ser rentable, al tiempo que reduce la proporción de pacientes que requieren TCT y disminuye la exposición a la radiación ${ }^{21}$.

\section{Equipo de respuesta para la embolia pulmonar}

Con el objetivo de brindar una mejor atención a los pacientes con EP se han creado y desarrollado los equipos de respuesta para la embolia de pulmón $(\text { EREP) })^{2,51}$. El primer EREP se creó en el 2012, en el Massachusetts General Hospital en Boston², y desde allí se han desarrollado en numerosos centros médicos de EE.UU., Europa y América del Sur ${ }^{51,52}$. El objetivo de un EREP es tener un solo equipo multidisciplinario de expertos en TEV que puedan responder rápidamente a los pacientes con EP aguda, ofrecer consultas e implementar un espectro completo de opciones terapéuticas $2,17,51-53$.

Inspirado en otros equipos de respuesta rápida (como los equipos de paro cardíaco, SCA con elevación del ST, accidente cerebrovascular, choque $)^{51-53}$, se basa en la evaluación acelerada multidisciplinaria (expertos en medicina vascular, emergentología, cuidados críticos, neumonología, imágenes, hemodinamia, hematología y cirugía vascular) de los pacientes de riesgo moderado-alto de EP, con el fin de elaborar un plan protocolizado basado en un correcto análisis de riesgos y beneficios $2,17,51-53$.

Si bien cada institución debe adaptar su equipo a sus necesidades y recursos disponibles, existen ciertos requisitos «mínimos» para tal equipo 2,17,51-53 (Fig. 3). Una de las definiciones de un EREP es un equipo multidisciplinario con base institucional que:

- Permite el acceso y tiene la capacidad para realizar una evaluación rápida y proporcionar opciones de tratamiento para un paciente con EP aguda $^{2,17,51-53}$.

- Posee un mecanismo formal para ejercer toda la gama de terapias médicas, quirúrgicas y endovasculares $2,17,51-53$.

- Puede proporcionar una evaluación y seguimiento adecuada de expertos ${ }^{51,53}$.

- Cuando sea posible, recopila, evalúa y envía datos sobre la efectividad del tratamiento brindado 2,17,51-53. 


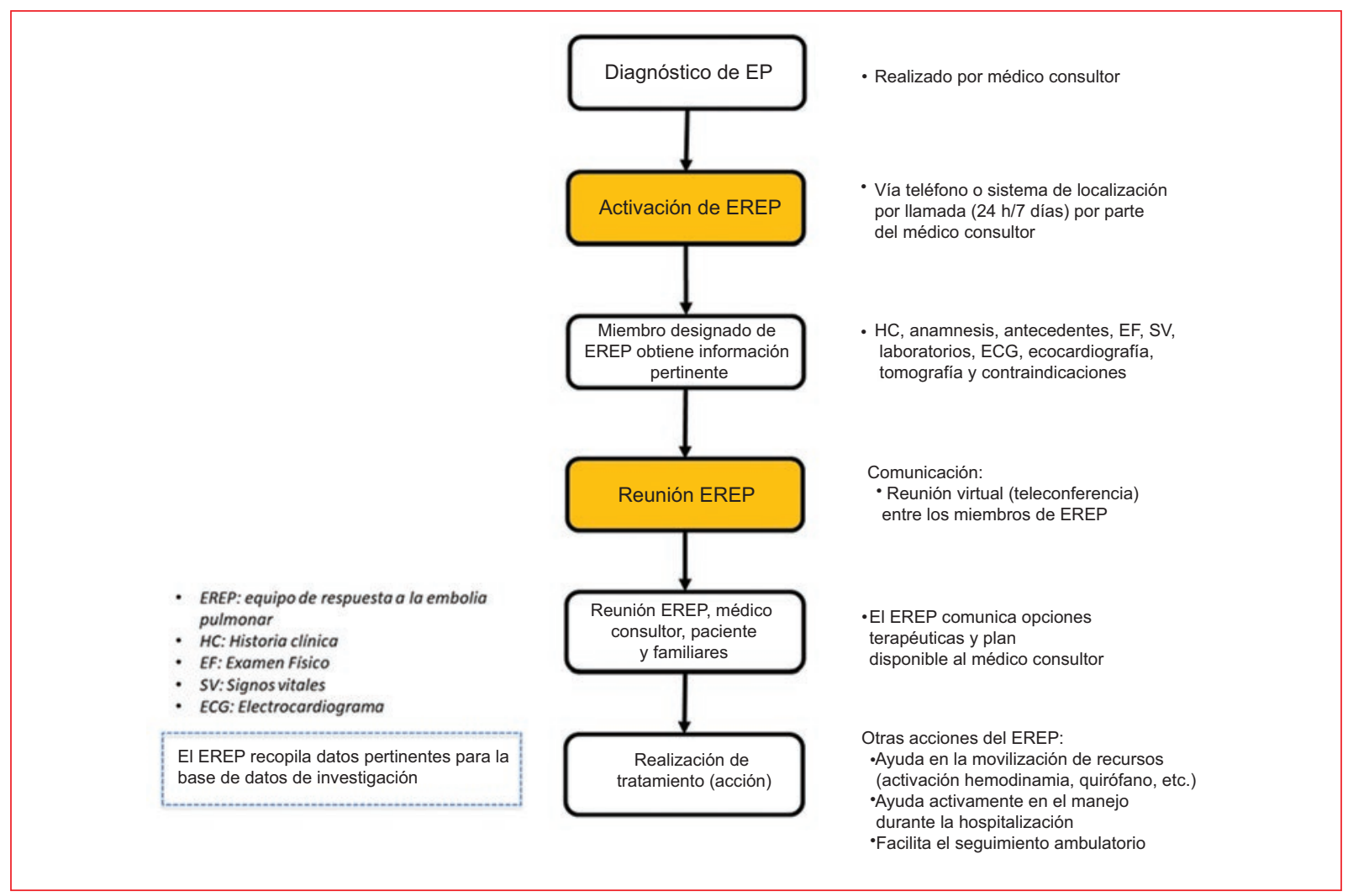

Figura 3. Algoritmo III. Diagrama de flujo esquemático del EREP que muestra la función dinámica, organizada y funcional de los miembros durante la atención de pacientes con EP compleja ladaptado de Porres-Aguilar $M$, Anaya-Ayala JE, Heresi GA, Rivera-Lebron BN. Pulmonary embolism response teams: A novel approach for the care of complex patients with pulmonary embolism. Clin Appl Thromb Hemost. 2018:1076029618812954)55.

EP: embolia pulmonar; EREP: equipo de respuesta a la EP; HC: historia clínica; EF: examen físico; SV: signos vitales; ECG: electrocardiograma.

\section{Conclusión}

En conclusión, el enfoque diagnóstico de la EP en el SE representa un verdadero desafío. Tener un alto grado de sospecha, conocer las características de los pacientes con sospecha de EP, y utilizar de forma criteriosa y combinada las diferentes herramientas diagnosticas permitirá hacer un diagnóstico precoz, con el consiguiente manejo eficiente del paciente.

\section{Financiamiento}

En este trabajo de investigación, no existió requerimiento de honorarios del investigador. El recurso tecnológico, informático y otros, estuvo disponible y al servicio por parte del investigador y no requirió renovación.

\section{Conflicto de intereses}

Este artículo de revisión es una versión actualizada que contiene fragmentos de un artículo publicado previamente en la Revista del Hospital El Cruce ${ }^{56}$ con autorización del autor y del editor jefe.

\section{Bibliografía}

1. Uresandi F, Monreal M, García-Bragado F, Domènech $P$, Lecumberri $R$, Escribano $\mathrm{P}$, et al. Consenso nacional sobre el diagnóstico, estratificación de riesgo y tratamiento de los pacientes con tromboembolia pulmonar. Arch Bronconeumol. 2013;49(12):534-47.

2. Dudzinski DM, Horowitz JM. Start-up, organization and performance of a multidisciplinary pulmonary embolism response team for the diagnosis and treatment of acute pulmonary embolism. Rev Esp Cardiol. 2017; 70(1):9-13.

3. Di Nisio M, van Es N, Büller HR. Deep vein thrombosis and pulmonary embolism. Lancet. 2016;388(10063):3060-73.

4. Konstantinides SV, Torbicki A, Agnelli G, Danchin N, Fitzmaurice D, Galiè N, et al. 2014 ESC guidelines on the diagnosis and management of acute pulmonary embolism. Eur Heart J. 2014;24(6):1-48.

5. Jimenez S. El diagnóstico del tromboembolia pulmonar: un reto permanente para el urgenciólogo. Emergencias. 2014;26:241-2.

6. Nazzareno G, Humbert M, Vachiery JL, Gibbs S, Lang I, Torbicki A, et al. Guía ESC/ERS 2015 sobre diagnóstico y tratamiento de la hipertensión pulmonar. Rev Esp Cardiol. 2016;69(1):177.e1-e62.

7. Giordano NJ, Jansson PS, Young MN, Hagan KA, Kabrhel C. Epidemiology, pathophysiology, stratification, and natural history of pulmonary embolism. Tech Vasc Interv Radiol. 2017;20(3):135-40.

8. Jarrett $\mathrm{H}$, Bashir R. Interventional management of venous thromboembolism: State of the Art. AJR. 2017;208(4):891-906.

9. Mazzolai L, Aboyans V, Ageno W, Agnelli G, Alatri A, Bauersachs R, et al. Diagnosis and management of acute deep vein thrombosis: a joint 
consensus document from the European society of cardiology working groups of aorta and peripheral vascular diseases and pulmonary circulation and right. Eur Heart J. 2017:1-13.

10. Sociedad Argentina de Cardiología. Consenso de enfermedad tromboembólica crónica, profilaxis y situaciones especiales. Rev Arg Cardiol. 2016;12(6):1-26.

11. Sociedad Argentina de Cardiología. Consenso de enfermedad tromboembólica aguda. Rev Argent Cardiol. 2016;8484(1):74-91.

12. Corrigan D, Prucnal C, Kabrhel C. Pulmonary embolism: the diagnosis, risk-stratification, treatment and disposition of emergency department patients Pulmonary embolism: the diagnosis, risk-stratification, treatment and disposition of emergency departmen. Clin Exp Emerg Med. 2016;3(3):117.

13. García-Frade Ruiz LF, Cuellar-Gamboa L. Actualidades en trombosis. Med Int Méx. 2016;32(1):103-14.

14. Tapson VF. Advances in the diagnosis and treatment of acute pulmonary embolism. F1000 Med Rep. 2012;11(May):1-11.

15. Jaff MR, McMurtry MS, Archer SL et al. Management of massive and submassive pulmonary embolism, iliofemoral deep vein thrombosis, and chronic thromboembolic pulmonary hypertension. A scientific statement from the American Heart Association Methods. Circulation. 2011; 123(16): $1788-830$

16. Schulman S. Update on the treatment of venous thromboembolism. Semin Thromb Hemost. 2016;42(8):891-8

17. Reza N, Dudzinski D. Pulmonary embolism response teams. Curr Treat Options Cardio Med. 2015:17-27.

18. Mcgee S. Evidence-based physical diagnosis. $4^{\text {th }}$ ed. Elsevier; 2018.

19. Renier W, Hoogma-von Winckelmann K, Verbakel JY et al. Signs and symptoms in adult patients with acute dyspnea: A systematic review and meta-analysis. Eur J Emerg Med. 2018;25(1):3-11.

20. Morice $A H$, Musset D, Samama MM et al. Task force report guidelines on diagnosis and management of acute. Eur Hear J. 2000;21(16):1301-36.

21. Sinclair A, Peprah K, Quay T et al. Optimal strategies for the diagnosis of acute pulmonary embolism: a health technology assessment - Project Protocol. Ottawa (ON): Canadian Agency for Drugs and Technologies in Health. 2018;6(3):1-413.

22. Ray $P$, Birolleau $S$, Lefort $Y$ et al. Acute respiratory failure in the elderly: etiology, emergency diagnosis and prognosis. Crit Care. 2006;10(3):1-12.

23. Crawford $F$, Andras $A$, Welch $\mathrm{K}$ et al. D-dimer test for excluding the diagnosis of pulmonary embolism (Review). Cochrane Database Syst Rev. 2016;(8):1-46.

24. Bozarth AL, Bajaj N, Wessling MR et al. Evaluation of the pulmonary embolism rule-out criteria in a retrospective cohort at an urban academic hospital. Am J Emerg Med. 2015;33(4):483-7.

25. Meyer G, Vicaut E, Danays $E$ et al. Fibrinolysis for patients with intermediate risk pulmonary embolism. N Engl J Med. 2014;4(370):1402-11.

26. Dachs RJ, Kulkarni D, Higgins LG. The pulmonary embolism rule-out criteria rule in a community hospital ED: A retrospective study of its potential utility. Am J Emerg Med. 2011;29(9):1023-7.

27. Wells PS, Anderson DR, Rodger $\mathrm{M}$ et al. Derivation of a simple clinical model to categorize patients probability of pulmonary embolism-increasing the models Ut. Thromb Haemost. 2000;83(3):416-20.

28. Wells PS, Anderson DR, Rodger MS et al. Excluding pulmonary embolism at the bedside without diagnostic imaging: Management of patients with suspected pulmonary embolism presenting to the emergency department by using a simple clinical model and D-dimer. Ann Intern Med. 2001;135:98-107.

29. Gibson NS, Söhne M, Kruip MJH. Further validation and simplification of the Wells clinical decision rule in pulmonary embolism. Thromb Haemost. 2008;99(1):229-34.

30. Chagnon I, Bounameaux H, Aujesky D et al. Comparison of two clinical prediction rules and implicit assessment among patients with suspected pulmonary embolism. Am J Med. 2002;113(4):269-75.

31. Le Gal G, Righini M, Roy PM et al. Prediction of pulmonary embolism in the emergency department: the revised Geneva score. Ann Intern Med 2006;144(3):165-71.

32. Kline JA, Mitchell AM, Kabrhel $C$ et al. Clinical criteria to prevent unnecessary diagnostic testing in emergency department patients with suspected pulmonary embolism. J Thromb Haemost JTH. 2004;2(8):1247-55.
33. Carpenter CR, Keim SM, Seupaul RA et al. Differentiating low-risk and no-risk PE patients: The PERC score. J Emerg Med. 2009;36(3):317-22.

34. Kline JA, Slattery D, Thompson BJ et al. Clinical features of patients with pulmonary embolism and a negative PERC rule result. Ann Emerg Med. 2013;61(1):122-4.

35. Lekerika NA, Arana-Arri EU, Montero AN y col. Probabilidad clínica de tromboembolia pulmonar:beneficio diagnóstico de las escalas de predicción y de los dímeros D. Emergencias. 2014;26(4):243-50.

36. Schouten HJ, Geersing GJ, Koek HL et al. Diagnostic accuracy of conventional or age adjusted D-dimer cut-off values in older patients with suspected venous thromboembolism: Systematic review and meta-analysis. BMJ. 2013;346(7910):1-13

37. Ortiz J, Saeed R, Little C et al. Age-adjusted D-dimer in the prediction of pulmonary embolism: Does a normal age-adjusted D-dimer rule out PE ? Biomed Res Int. 2017:10:1-7.

38. Chaaya G, Vishnubhotla P. Pulmonary vein thrombosis: A recent systematic review. Cureus. 2017;9(1):1-13.

39. McHugh KB, Visani L, DeRosa M et al. Gender comparisons in pulmonary embolism (results from the International Cooperative Pulmonary Embolism Registry [ICOPER]). Am J Cardiol. 2002:89(5):616-9.

40. De la Quintana Gordon FB, Nacarino Alcorta B. Ecografía pulmonar básica. Parte 1. Ecografía pulmonar normal y patología de la pared torácica y la pleura. Rev Esp Anestesiol Reanim. 2015;62(6):322-36.

41. Blanco SR, Gaforio AF, Galán BH, et al. Utilidad de la ecografía clínica en el diagnóstico del paciente con disnea. Galicia Clínica. 2017;77(4):161.

42. De la Quintana Gordon FB, Nacarino Alcorta B, Fajardo Pérez M. Ecografía pulmonar básica. Parte 2. Patología parenquimatosa. Rev Esp Anestesiol Reanim. 2015;62(6):337-49.

43. Lichtenstein DA, Mezière GA. Relevance of lung ultrasound in the diagnosis of acute respiratory failure the BLUE protocol. Chest. 2008;134(1): 117-25.

44. Lichtenstein DA. BLUE-Protocol and FALLS-Protocol. Chest. 2015; 147(6):1659-70.

45. Lichtenstein D. Fluid administration limited by lung sonography: The place of lung ultrasound in assessment of acute circulatory failure (the FALLS-protocol). Expert Rev Respir Med. 2012;6(2):155-62.

46. Squizzato A, Rancan E, Dentali E et al. Diagnostic accuracy of lung ultrasound for pulmonary embolism: a systematic review and meta-analysis. J Thromb Haemost. 2013;11(7):1269-78

47. Jiang $L, M a Y$, Zhao $C$ et al. Role of transthoracic lung ultrasonography in the diagnosis of pulmonary embolism: A systematic review and meta-analysis. PLoS One. 2015;10(6):e0129909.

48. Bajaj $A$, Rathor $P$, Sehgal $V$ et al. Prognostic value of biomarkers in acute non-massive pulmonary embolism: A systematic review and meta-analysis. Lung. 2015;193(5):639-51.

49. Zhou $\mathrm{M}, \mathrm{Hu} Y$, Long $X$. Diagnostic performance of magnetic resonance imaging for acute pulmonary embolism: a systematic review and meta-analysis. J Thromb Haemost. 2015;13(9):1623-34.

50. Feng $\mathrm{C}, \mathrm{Yi}$-Hong $\mathrm{S}$, Xu-Qing $\mathrm{S}$ et al. Comparison between $\mathrm{CT}$ and $\mathrm{MRI}$ in the assessment of pulmonary embolism. Medicine (Baltimore). 2017; 96(52):1-7.

51. Dudzinski DM, Piazza G. Multidisciplinary pulmonary embolism response teams. Circulation. 2016:133(1):98-103.

52. Rosovsky R, Chang $Y$, Rosenfield $K$ et al. Changes in treatment and outcomes after creation of a pulmonary embolism response team (PERT), a 10-year analysis. J Thromb Thrombolysis. 2019:47(1):31-40.

53. Rodriguez-Lopez J, Channick R. The Pulmonary Embolism Response Team: What is the ideal model? Semin Respir Crit Care Med. 2017;38(1):51-5.

54. Stein PD, Woodarad PK, Weg JG, Wakefield TW, Tapson VF, Sostman HD, et al.; PIOPED II Investigators. Diagnostic pathways in acute pulmonary embolism: Recommendations of the. Radiology. 2007; 2(1):15-21.

55. Porres-Aguilar M, Anaya-Ayala JE, Heresi GA, Rivera-Lebron BN. Pulmonary embolism response teams: A novel approach for the care of complex patients with pulmonary embolism. Clin Appl Thromb Hemost. 2018: 1076029618812954

56. García D. Embolia pulmonar en el servicio de emergencias: Parte 1. Rev. Hosp. El Cruce 2018;(22):14-27. 